\title{
SUBJECT INDEX - Volume 9
}

International Journal on Energy for a Clean Environment

Page Numbers for Issues:

Issues 1-3, 1-227

Abanades, J.C., 91

Altendorfner, M., 211

Anthony, E.J., 91

Borissov, A.A., 159

Carvalho, L., 127

Chudnovsky, Y., 103

Dannehl, M., 211

Fedchenia, I., 143

Gillan, L., 47

Gorbounov, M., 143

Gorski, J., 1

Issendorff, F.V., 211

Kozlov, A., 103
Lu, D., 91

Lundgren, J., 127

Marvin, D., 143

Nosach, V.G., 39, 119, 223

Rogers, K., 143

Salvador, C., 91

Scheffknecht, G., 179

Shraiber, A.A., 39, 119, 223

Shtern. V.N., 159

Soroka, B., 65, 195

Viskanta, R., 13

Wopienka, E., 127

Yantovsky, E., 1 


\section{SUBJECT INDEX - Volume 9 \\ International Journal on Energy for a Clean Environment \\ Page Numbers for Issues: \\ Issues 1-3, 1-227}

agricultural fuels, 127

ash, 127

CFD, 143

$\mathrm{CO}_{2}$ capture, 91

$\mathrm{CO}_{2}$ emissions, 179

carbon capture, 179

clean energy, 1

coanda effect, 195

combined $\mathrm{CO} 2$ cycles, 1

combustion, 65, 127

combustion devices, 13

combustion stability, 195

dew point, 47

dimpled tube, 103

effectiveness, 47

efficiency, 39, 119, 223

emissions, 127

energy supply systems, 1

energy-using efficiency, 65

evaporative cooling, 47

excess air ratio, 211

FEA, 143

finned tube, 103

flame aerodynamics, 195

flat-flame burner (FFB), 195

flue gas recirculation, 159

fluid bed combustion, 91

fossil fuel, 179

fouling

gas furnace, 195

gas-turbine plant, 119, 223

harmful emissions, 39, 119, 223

heat transfer enhancement, 103

high-pressure combustion, 211

IGBT, 143 industrial furnace, 65

low-emission combustion, 195

maisotsenko cycle, 47

meridional circulation, 159

mitigation, 103

modeling, 13

nanoparticles, 211

NOx and CO formation, 195

particle trajectories, 159

pollutant emissions, 13

porous burners, 13

porous inert materials, 13

porous inert media, 211

power electronics, 143

power plant technology, 179

pressure drop reduction, 103

reactivation, 91

recirculation of combustion products, 195

reformed fuel, 39

slagging, 127

specific emission of NOx (CO2), 65

specific fuel consumption, 65

state space model, 143

steam-and-gas plant, 39

thermal management, 143

thermal transient response, 143

thermochemical and air recuperation, 119

thermochemical recuperation, 39

thermochemical, steam and air recuperation, 223

thermodynamic equilibrium, 65

velocity profile, 195

vortex chamber, 159

wet bulb, 47

zero-emission power plant, 179 